\title{
Optimal Size of a Complex Network
}

\author{
H. Hong* \\ Korea Institute for Advanced Study, Seoul 130-012, Korea \\ Beom Jun Kim \\ Department of Molecular Science and Technology, Ajou University, Suwon 442-749, Korea \\ M.Y. Cho H \\ Department of Physics, Seoul National University, Seoul 151-747, Korea
}

\begin{abstract}
We investigate the response behavior of an Ising system, driven by an oscillating field, on a smallworld network, with particular attention to the effects of the system size. The responses of the magnetization to the driving field are probed by means of Monte Carlo dynamic simulations with the rewiring probability varied. It is found that at low and high temperatures the occupancy ratio, measuring how many spins follow the driving field, behaves monotonically with the system size. At intermediate temperatures, on the other hand, the occupancy ratio first grows and then reduces as the size is increased, displaying a resonance-like peak at a finite value of the system size. In all cases, further increase of the size eventually leads to saturation to finite values; the size at which saturation emerges is observed to depend on the temperature, similarly to the correlation length of the system.
\end{abstract}

PACS numbers: $89.75 . \mathrm{Hc}, 75.10 . \mathrm{Hk}$

It has been known that under appropriate circumstances, the presence of noise in a driven system can enhance rather than suppress the output of the system. Such attractive phenomena, called stochastic resonance (SR), have been widely investigated in various systems of many practical applications [1, 2]. Recently, those SR phenomena have also been examined in the context of the system size resonance: Stochastic flips of the mean field are observed to depend on the system size, leading the linear response of the system to reach a maximum at a certain system size [3]. Those works considered two systems of fully coupled noisy oscillators and one twodimensional Ising system with nearest neighbor interactions. Namely, the underlying connection topology of dynamic variables was assumed to be regular, with either local or global connections. Meanwhile, recent studies of neuronal networks, computer networks, biochemical networks, and even social networks, have revealed that various real systems in nature possess quite complex structures, which can be described neither by regular networks nor by completely random networks [4]. Accordingly, it is desirable to study effects of the system size on collective responses in the systems with the connection topology of complex networks, which can be made more realistic. In particular, the interplay between the system size and noise may be relevant in various biological systems such as neural networks and other cell networks, which consist of finite numbers of elements. For example, in the study of stochastic resonance in biological systems, optimal sizes of calcium ion channel clusters have been

\footnotetext{
*Electronic address: hhong@kias.re.kr

${ }^{\dagger}$ Also at Korea Institute for Advanced Study, Seoul 130-012, Korea.
}

examined. Observed is that the clustering of the release channels in small clusters increases the sensitivity of the calcium response [5]. This suggests a possible realization of the system, providing motivation for the investigation of the size resonance in the complex network structure.

In this paper we consider an Ising model on a complex network, specifically, on the Watts and Strogatz (WS) type small-world network [6]. It is well known that the WS network is characterized by a small characteristic path length $\ell \sim \ln N$, where $N$ is the number of nodes constituting the network, and a large clustering coefficient. Both are commonly observed properties of real networks in nature. The WS network in this paper is constructed following Ref. [6]: A regular one-dimensional (1D) network of $N$ nodes is first constructed with local connections of range $k$, under periodic boundary conditions. At this stage each node on the network has $2 k$ nearest neighbors. Next, each local link is visited once and, with the rewiring probability $P$, removed and reconnected to a randomly chosen node. After a whole sweep of the entire network, the average number of shortcuts in the network of size $N$ is given by $N P k$. Accordingly, the rewiring probability $P$ may be regarded as the fraction of the average number of shortcuts over the total number of connections $N k$. In this paper, the local interaction range $k$ is set equal to two for convenience; longer ranges $(k>2)$ are not expected to lead to any qualitative difference. After the WS network is built as above, an Ising spin is put on every node, and an edge (or a link) connecting two nodes is regarded as the coupling between the two spins at the two nodes. Finally, we apply an oscillatory field, driving the Ising spins, and the corresponding responses of the average spin, i.e., the magnetization are probed via Monte Carlo dynamic simulations, with attention to the effects of the system size. 
The Hamiltonian for the field-driven Ising model on the WS network, which is constructed as described above, reads

$$
H=-\frac{1}{2} \sum_{i, j} J_{i j} \sigma_{i} \sigma_{j}-h(t) \sum_{i} \sigma_{i}
$$

where the ferromagnetic spin-spin interaction strength $J_{i j}$ is given by

$$
J_{i j}=J_{j i} \equiv \begin{cases}J & \text { for } j \in \Lambda_{i} \\ 0 & \text { otherwise }\end{cases}
$$

The neighborhood $\Lambda_{i}$ of node $i$ stands for the set of nodes connected to $i$ (via either local edges or shortcuts), and $\sigma_{i}(= \pm 1)$ represents the Ising spin at node $i$. The sinusoidally oscillating magnetic field $h(t)=h_{0} \cos \Omega t$ is applied with the driving amplitude $h_{0}$ and frequency $\Omega$, while the system is assumed to be in contact with an isothermal heat bath at temperature $T$. We probe the dynamics of the system described by Eq. (11) by means of Monte Carlo (MC) dynamic simulations, employing the heat bath single spin-flip algorithm [7] and measuring the time $t$ in units of the MC time step. For thermalization, we start from sufficiently high temperatures and lower the temperature $T$ slowly with the decrement $\Delta T=0.01$ (in units of $J / k_{B}$ with the Boltzmann constant $k_{B}$ ). The driving amplitude and frequency are taken to be $h_{0}=0.1$ and $\Omega=0.001$. We have also considered different frequencies, for example, $\Omega=0.01$ and 0.1 , and found that the resonance-like peak indicating the system size resonance behavior tends to diminish at higher frequencies (see below). While simulations are performed at a given temperature, the data from the first $10^{5} \mathrm{MC}$ steps are discarded, which turns out to be sufficient for stationarity, and measurements are made for next $10^{5} \mathrm{MC}$ steps. Networks of various sizes are constructed as described above, and averages are taken over 100 different network realizations for each size.

To investigate the collective response of the system, we measure the occupancy ratio $R$ which is defined to be the average fraction of the spins in the direction of the external field [8, 9]:

$$
R \equiv\left\langle\frac{\text { number of spins in the direction of } h(t)}{\text { total number of spins }}\right\rangle,
$$

where $\langle\cdots\rangle$ denotes the time average. In other words, $R$ measures how many spins follow the oscillating magnetic field. It is easy to understand that $R$ approaches the value $1 / 2$ in both low- and high-temperature limits (see, e.g., Ref. [9, 10]) and becomes increased near the stochastic resonance temperature, reflecting that more spins follow the external driving. Such SR phenomena have been observed in the system of given size, and it has been demonstrated that the matching condition of two time scales, the relaxation time of the system and the inverse frequency of the driving field, yields the optimal noise strength $T_{S R}$ at which the system displays maximum responses 9, 10]. Here we consider the system studied in Ref. 10 from a different point of view and examine the behavior of the occupancy ratio with the system size at various temperatures, probing the size resonance.

In the absence of long-range shortcuts $(P=0)$, the network structure reduces to that of the 1D regular network with only local couplings of range $k$. Accordingly, when $P=0$, the field-driven Ising model described by Eq. (11) as well as the undriven model $[h(t)=0]$ does not exhibit long-range order at finite temperatures, yielding $T_{c}=0$. Note, however, that even such a 1D system displays SR behavior at finite temperatures 11]. In the presence of long-range shortcuts $(P \neq 0)$, on the other hand, it has been found that both driven and undriven Ising model display ferromagnetic order at finite temperatures 10, 12]; furthermore, double SR peaks, which originate from matching of two time scales have been observed.

We first consider the case without long-range shortcuts $(P=0)$, i.e., the purely 1D system, and show in Fig. 1 the behavior of the occupancy ratio $R$ versus the system size $N$ at various temperatures. It is observed that the occupancy ratio $R$ first increases and eventually saturates to a finite value as the system size $N$ is increased. The saturation size $N_{s}$, beyond which the occupancy ratio $R$ does not increase any more, reduces as the temperature $T$ is raised. Figure 2 displays such temperature dependence of the saturation size $N_{s}$, which has been taken as the size giving the occupancy ratio with the difference from the stationary value less than $5 \times 10^{-4}$. One can observe the exponential behavior: $N_{s} \propto e^{c / T}$ with $c=5.6 \pm 0.7$, which is reminiscent of the behavior of the correlation length. In the 1D Ising model the correlation length $\xi$ diverges in the low-temperature limit as $\xi \sim e^{k(k+1) / T}[12]$. Here the local interaction range $k$ has been chosen to be two $(k=2)$, leading to the behavior $\xi \sim e^{6 / T}$, essentially the same as that of the saturation size. It is thus concluded that the saturation behavior emerges when the system size reaches the correlation length of the system.

Meanwhile, in the presence of long-range shortcuts $(P \neq 0)$, substantially different behavior has been obtained for the occupancy ratio. In Fig. 3 the occupancy ratio $R$ of the system with the rewiring probability $P=0.5$ is displayed as the system size $N$ is varied. At low temperatures $(T \lesssim 1.5), R$ first decreases monotonically with the size $N$ and then saturates to the value 0.5 ; at high temperatures $(T \gtrsim 2.9)$, on the contrary, $R$ increases monotonically to the saturation value larger than 0.5 , depending on the temperature. In contrast to these monotonic behaviors, at intermediate temperatures $(1.5 \lesssim T \lesssim 2.9)$, the occupancy ratio $R$ behaves nonmonotonically, exhibiting a maximum at a finite value of the system size $N$. The height of such a resonancelike peak tends to increase as the temperature is raised. We have also considered different values of the rewiring probability $P$ as well as of the driving frequency $\Omega$. It is found that as $P$ is increased, the range of the temper- 


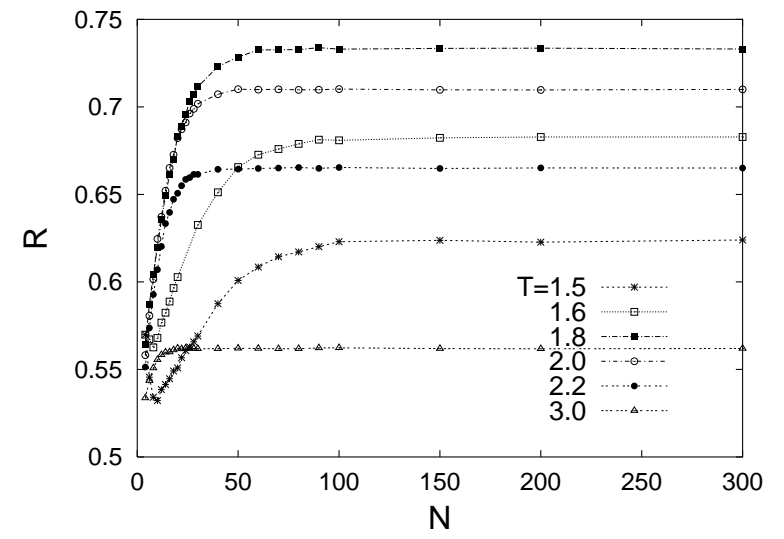

FIG. 1: Behavior of the occupancy ratio $R$ with the system size $N$ at various temperatures $T$ in the absence of long-range shortcuts. Lines are merely guides to eyes.

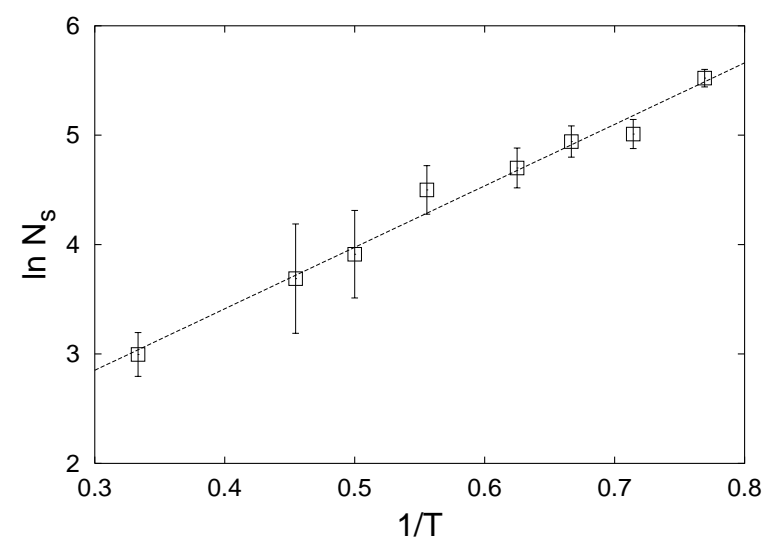

FIG. 2: Behavior of the saturation size $N_{s}$ with the temperature $T$ in the absence of shortcuts, exhibiting a linear relation between $\ln N_{s}$ and $1 / T$, with the slope $c=5.6 \pm 0.7$. The dotted line, obtained by the least-square fit, represents $\ln N_{s}=5.6 / T+1.2$.

ature, in which the size-resonance behavior is displayed, becomes wider and that the saturation temperature beyond which $R$ shows saturation behavior increases. On the other hand, as the driving frequency is increased, the position of the resonance peak shifts toward smaller values of the system size, thus tending to yield monotonic decrease of $R$.

Note that this resonance behavior manifests two kinds of length scale in the system with long range shortcuts: the saturation size $N_{s}$ and the resonance size $N_{m}$ at which $R$ reaches the maximum. To understand the possible relation with the correlation length even in the presence of long range shortcuts, we examine the behaviors of $N_{s}$ and $N_{m}$, which are displayed in Figs. 4- 6

Figure 4 exhibits the saturation size $N_{s}$ versus the temperature $T$ in the system with $P=0.5$. For comparison with the correlation volume described by $\xi_{V} \sim$ $\left|T-T_{c}\right|^{-\bar{\nu}}[13]$, the data points are plotted in the log-

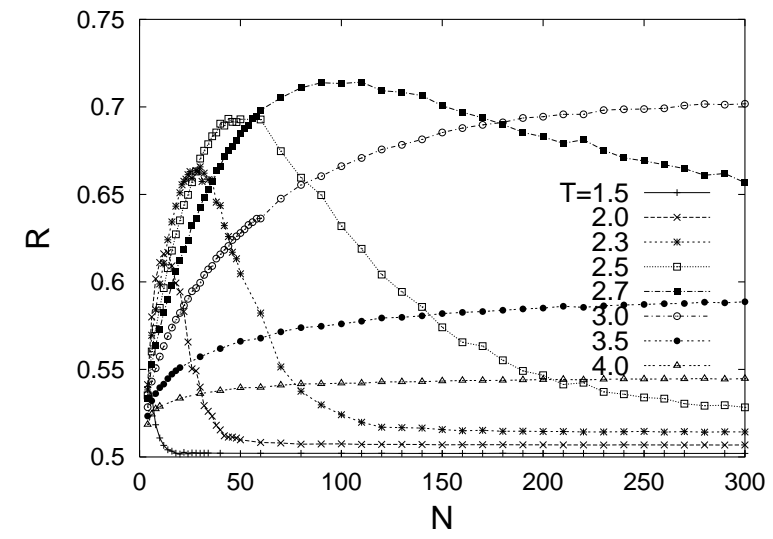

FIG. 3: The occupancy ratio $R$ vs the system size $N$ in the presence of the long-range shortcuts $(P=0.5)$ at various temperature $T$. Lines are merely guides to eyes.

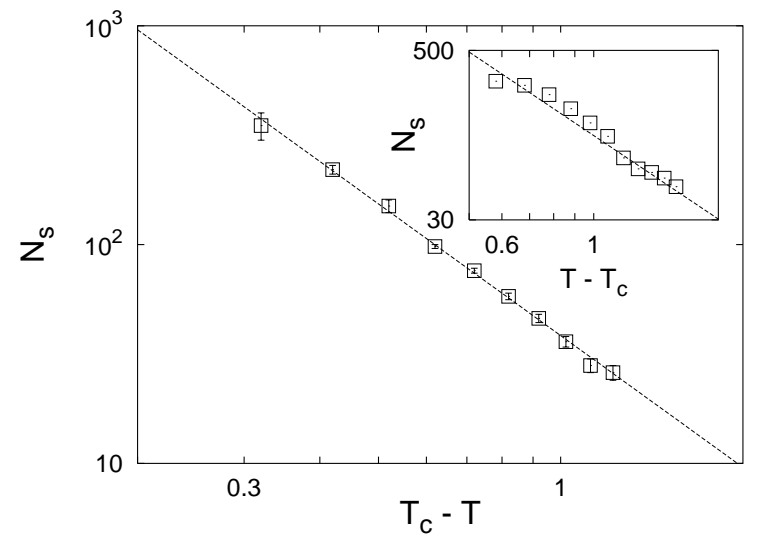

FIG. 4: Behavior of the saturation size $N_{s}$ with the temperature $T$ for $P=0.5$ at low temperatures $\left(T<T_{c}\right)$, where the value $T_{c}=2.7$ obtained from the fitting has been used. The least-square fit represented by the dashed line corresponds to $\log N_{s}=-2.0 \log \left(T_{c}-T\right)+1.6$, the slope of which gives $\bar{\nu}=2$.0. Inset: Behavior of $N_{s}$ at temperatures higher than $T_{c}$. The dashed line, obtained from the least-square fit, is given by $\log N_{s}=-2.0 \log \left(T-T_{c}\right)+2.1$, where the slope again leads to $\bar{\nu}=2.0$. The error bars have sizes not larger than the symbol size.

arithmic scale, thus fitted to a linear relation between $\ln N_{s}$ and $\ln \left|T-T_{c}\right|$ with the proportionality constant (slope) $\bar{\nu}$. From this fitting, we obtain $T_{c} \approx 2.7$, with which the slope is estimated to be $\bar{\nu}=2.0 \pm 0.1$. Noting that in $d$ dimensions, the correlation volume relates with the correlation length $\xi$ via $\xi_{V} \sim \xi^{d}$ and the behavior $\xi \sim\left|T-T_{c}\right|^{-\nu}$, we thus have $\bar{\nu}=d \nu$ in a $d$-dimensional system. Here it is known that the (effective) dimension $d$ of a mean-field system should be taken as the uppercritical dimension $d_{u}$ [14], leading to $\bar{\nu}=d_{u} \nu$. With $d_{u}=4$ and $\nu=\nu_{M F}=1 / 2$ for a mean-field system, we conclude that the value $\bar{\nu}=2.0$ indicates a transition of the mean-field nature [13]. We also examine the other length scale $N_{m}$ and show its temperature dependence in 


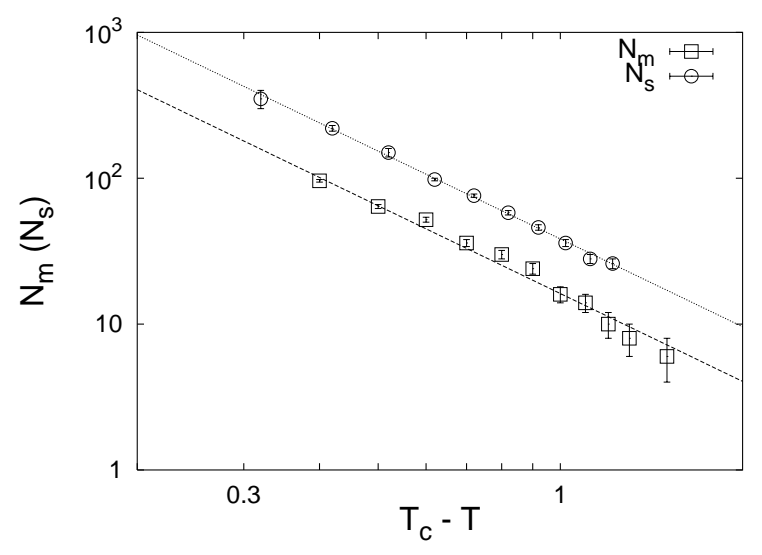

FIG. 5: The resonance size $N_{m}$ together with the saturation size $N_{s}$ vs the temperature $T$ in the presence of shortcuts $(P=0.5)$. The values of $T_{c}$, obtained from the fitting, are given by 3.1 and 2.7 for $N_{m}$ and $N_{s}$, respectively. The dotted line represents the corresponding least-square fit of $N_{m}$, which is described by $\log N_{m}=-2.0 \log \left(T_{c}-T\right)+1.2$. Thus both $N_{m}$ and $N_{s}$ result in the same value $\bar{\nu}=2.0$.

Fig. [5] where for convenience $N_{s}$, shown already in Fig.4 is also plotted. It is shown that both the two length scales behave similarly with the temperature, yielding essentially the same value of the exponent $\bar{\nu}=2.0 \pm 0.1$ (see the slopes of the two fitted lines). Accordingly, both the two length scales $N_{s}$ and $N_{m}$ apparently measure the correlation length of the system. On the other hand, the fitting parameter $T_{c}$ for $N_{m}$ turns out to be 3.1, which is somewhat higher than the value 2.7 obtained from fitting of $N_{s}$. At this stage it is difficult to discern unambiguously the difference; more extensive simulations and careful analysis are necessary for confirming the origin as well as the presence of this discrepancy.

Finally, we consider systems with different rewiring probabilities, and examine how the rewiring probability $P$ affects the resonance behavior. Figure [ $[$ shows the behavior of the resonance size $N_{m}$ with the temperature at various values of $P$. It is observed that $N_{m}$ first increases slowly with the temperature $T$ then very fast as $T$ approaches $T_{c}$, which again reminds us of the behavior of the correlation length. Behavior of $N_{m}$ with the rewiring probability $P$ at given temperature $T=2.2$ is displayed in the inset of Fig. 6] Note the rather fast decrease of $N_{m}$ for small rewiring probabilities $(P \lesssim 0.5)$ and the saturation behavior for large rewiring probabilities $(P \gtrsim 0.5)$. Such saturation behavior has also been reported in the synchronization of the system of coupled oscillators on a WS network [15]. It is also noteworthy that $N_{m}$ decreases as shortcuts are introduced to the system; the shortcuts tend to decrease the optimal system size which corresponds to the maximum collective response at given temperature and rewiring probability.

In conclusion, we have investigated the effects of the system size on the collective response, measured by the occupancy ratio, in the oscillatory field-driven Ising

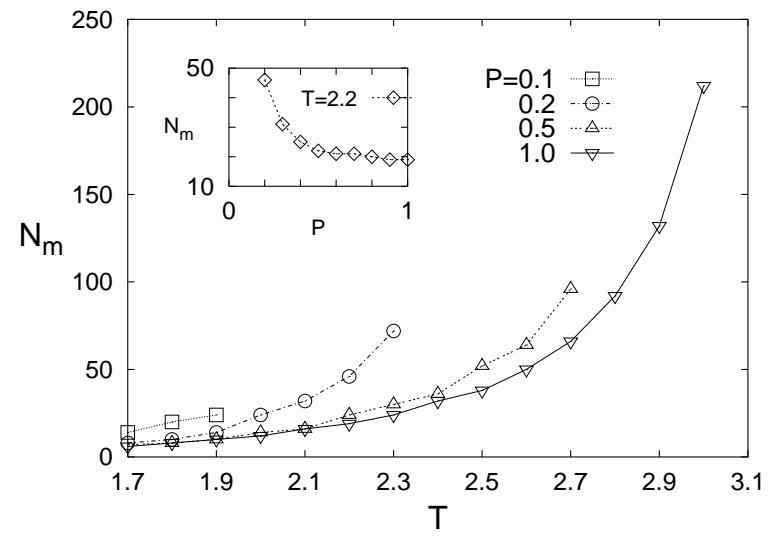

FIG. 6: The resonance size $N_{m}$ vs the temperature $T$ at various values of the rewiring probability $P$. Inset: Behavior of $N_{m}$ with the rewiring probability $P$ at temperature $T=2.2$.

model on WS networks. In the purely one-dimensional system without long-range interactions, which does not undergo a phase transition at any finite temperature, the occupancy ratio has been found to display monotonic behavior, not exhibiting a resonance peak. As long-range interactions come into the system, on the other hand, system-size resonance, characterized by nonmonotonic behavior, has been observed to emerge, thus suggesting a possible relation between the size resonance and a finite-temperature phase transition. The resonance size at which the occupancy ratio reaches the peak may be regarded as the optimal size of the network, in view of the maximum response. It is noteworthy that the optimal size as well as the saturation size displays temperaturedependent behavior, which is essentially the same as that of the correlation length of the system. This suggests the interesting possibility of estimating the correlation length from the stochastic resonance behavior at various sizes. Namely, the size resonance phenomena may be used as a tool to measure the correlation length. Note also that at given temperature both length scales, the optimal size and the saturation size, tend to decrease as the amount of long-range interactions is increased.

As a possible application of the system size resonance, we suggest biological systems such as the assembly of beta cells which reside in the islets of Langerhans in a pancreas 16]. It is known that the beta cells form clusters, each with a finite number of cells, rather than gathering together as one unit. Thus speculated is the possibility that the function of the beta cells may be optimized via the mechanism of the system-size resonance. In addition, the system size resonance behavior may also be useful for understanding the formation of the public opinion [17. 


\section{Acknowledgments}

H.H. thanks J. Lee for providing the privilege of using the computing facility Gene. B.J.K. was supported in part by the Korea Science and Engineering Founda- tion through Grant No. R14-2002-062-01000-0. M.Y.C. thanks the Korea Institute for Advanced Study for hospitality during his visit, where this work was performed, and acknowledges the partial support from the SwissKorean Outstanding Research Efforts Award Program.
[1] B. McNamara and K. Wiesenfeld, Phys. Rev. A 394854 (1989); P. Jung and P. Hänggi, ibid. 44, 8032 (1991); L. Gammaitoni, P. Hänggi, P. Jung, and F. Marchesoni, Rev. Mod. Phys. 70, 223 (1998).

[2] S. Fauve and F. Heslot, Phys. Lets. A 97, 5 (1983); M.H. Devoret, J.M. Martinis, D. Esteve, and J. Clarke, Phys. Rev. Lett. 53, 1260 (1984); B. McNamara, K. Wiesenfeld and R. Roy, ibid. 60, 2626 (1988).

[3] A. Pikovsky, A. Zaikin, and M.A. de la Casa, Phys. Rev. Lett. 88, 050601 (2002); R. Toral, C.R. Mirasso, and J.D. Gunton, e-print cond-mat/020755.

[4] For reviews of complex networks, see, e.g., Science 284, 79-109 (1999); M.E.J. Newman, J. Stat. Phys. 101, 819 (2000); S.H. Strogatz, Nature 410, 268 (2001); R. Albert and A.-L. Barabási, Rev. Mod. Phys. 74, 47 (2002); S.N. Dorogovtsev and J.F.F. Mendes, Adv. Phys. (in press).

[5] G. Schmid, I. Goychuk, and P. Hänggi, Europhys. Lett. 56, 22 (2001); P. Jung and J.W. Shuai, ibid. 56, 29 (2001); J.W. Shuai and P. Jung, Phys. Rev. Lett. 88, 068102 (2002).

[6] D.J. Watts and S.H. Strogatz, Nature (London) 393, 440 (1998).

[7] See, e.g., M.E.J. Newman and G.T. Barkema, Monte
Carlo Methods in Statistical Physics (Oxford, New York, 1999).

[8] J.F. Lindner, B.K. Meadows, W.L. Ditto, M.E. Inchiosa, and A.R. Bulsara, Phys. Rev. Lett. 75, 3 (1995).

[9] B.J. Kim, P. Minnhagen, H.J. Kim, M.Y. Choi, and G.S. Jeon, Europhys. Lett. 56, 333 (2001).

[10] H. Hong, Beom Jun Kim, and M.Y. Choi, Phys. Rev. E 66, 011107 (2002).

[11] J.J. Brey and A. Prados, Phys. Lett. A 216, 240 (1996).

[12] M. Gitterman, J. Phys. A: Math. Gen. 33, 8373 (2000); A. Barrat and M. Weigt, Eur. Phys. J. B 13, 547 (2000).

[13] B.J. Kim, H. Hong, P. Holme, G.S. Jeon, P. Minnhagen, M.Y. Choi, Phys. Rev. E 64, 056135 (2001).

[14] R. Botet, R. Jullien, and P. Pfeuty, Phys. Rev. Lett. 49, 478 (1982).

[15] H. Hong, M.Y. Choi, and B.J. Kim, Phys. Rev. E 65, 026139 (2002).

[16] See, e.g., H.D. Patton, A.F. Fuchs, B. Hille, A.M. Scher, and R. Steiner, Textbook of Physiology (Saunders, Philadelphia, 1989).

[17] P. Babinec, Phys. Lett. A 225, 179 (1997). 